\title{
Multisoliton Solutions and Breathers for the Coupled Nonlinear Schrödinger Equations via the Hirota Method
}

\author{
Ting-Ting Jia, Yu-zhen Chai, and Hui-Qin Hao \\ School of Mathematics, Taiyuan University of Technology, Taiyuan 030024, China \\ Correspondence should be addressed to Hui-Qin Hao; math0351@sina.com
}

Received 18 January 2016; Accepted 9 March 2016

Academic Editor: Hassan Askari

Copyright (C) 2016 Ting-Ting Jia et al. This is an open access article distributed under the Creative Commons Attribution License, which permits unrestricted use, distribution, and reproduction in any medium, provided the original work is properly cited.

Under investigation in this paper are the coupled nonlinear Schrödinger (CNLS) equations with dissipation terms by the Hirota method, which are better than the formal Schrödinger equation in eliciting optical solitons. The bilinear form has been constructed, via which multisolitons and breathers are derived. In particular, the three-bright soliton solution and breathers are derived and simulated via some pictures. The propagation characters are analysed with the change of the parameters.

\section{Introduction}

Since predicted and demonstrated experimentally, the propagation of temporal soliton envelopes in nonlinear optical media has attracted a great deal of attention due to the applications in the optical fiber long-distance transmission systems [1-7]. In the picosecond regime, optical solitons are based on the balance between the group velocity dispersion and self-phase modulation [8]. The dynamics of such solitons are described by the nonlinear Schrödinger (NLS) equation $[9,10]$

$$
i u_{t}+\frac{1}{2} u_{x x}+|u|^{2} u=0
$$

where $u$ denotes the slowly varying complex envelope of the wave and $x, t$ depend on the longitudinal distance and retarded time, respectively. Taking account of third-order dispersion, self-steepening, and other nonlinear effects, by Darboux transformation [11] described the rogue waves and rational solutions of the Hirota equation in the following form:

$$
i u_{t}+\alpha\left(2|u|^{2} u+u_{x x}\right)+i \beta\left(u_{t t t}+6|u|^{2} u_{t}\right)=0,
$$

where $\alpha=1 / 2$ and the two terms in (2) that enter with a real coefficient $\beta$ are responsible for the third-order dispersion and a time-delay correction to the cubic term. For the further study of the higher-order terms and nonlinear terms influence on soliton propagation mechanism, Guo have constructed the Lax Pair through Darboux transformation and derived the breathers and multisoliton solutions for the fourth-order generalized nonlinear Schrödinger equation as follows [12]:

$$
\begin{aligned}
i u_{t} & +u_{x x}+2|u|^{2} u+\tau\left(u_{x x x x}+8|u|^{2} u_{x x}+2 u^{2} u_{x x}^{*}\right. \\
& \left.+6 u^{*} u_{x}^{2}+4\left|u_{x}\right|^{2} u+6|u|^{4} u\right)=0 .
\end{aligned}
$$

Even though (1), (2), and (3) may adequately describe the propagation in a single-mode waveguide, switching operations, and routing, other optical effects which involve soliton pulses require the interaction between two or more modes [13, 14]. For several past decades, soliton propagation in the coupled nonlinear Schrödinger (CNLS) equations with different effective terms has been investigated from numerical simulation through applying Painlevé analysis [14], constructing Lax pair [15, 16], and carrying on gauge transformation [17-19]. In particular, [20] reveals the ABand Ma-breathers and localized solitons for the HirotaMaxwell-Bloch system on constant backgrounds in erbium doped fibers in detail. Recently, many solutions including bright solitons, bounded solitons, and collisions between 
two solitons have been derived via Darboux transformation $[21,22]$ and Bäcklund transformation $[23,24]$. The bound states with periodic attraction and repulsion between two solitons have been derived by the Lax Pair in [25], which are contained in the coupled higher-order nonlinear Schrödinger equations with variable coefficients. Subsequently, multidimensional coupled nonlinear equations attract the attention of so many science researchers by a variety of methods [26-28]. References [29, 30], especially, have investigated the $(2+1)$-dimensional coupled nonlinear Schrödinger equations via the Hirota method and analysed two-solitons and collisions between solitons in detail. Considering the above, we investigate the following CNLS equations as the form [6]

$$
\begin{aligned}
i u_{x} & +c_{1} u_{t t}+2\left(\alpha|u|^{2}+\beta|v|^{2}\right) u+\left(\Delta_{1}+\Delta_{2}\right) u \\
& +\left(K_{1}+i K_{2}\right) v=0, \\
i v_{x} & +c_{2} v_{t t}+2\left(\beta|u|^{2}+\gamma|v|^{2}\right) v+\left(\Delta_{1}-\Delta_{2}\right) u \\
& +\left(K_{1}-i K_{2}\right) v=0,
\end{aligned}
$$

where $c_{1}$ and $c_{2}$ are the group velocity coefficients, $\alpha, \beta$, and $\gamma$ are the parameters related to nonlinear effects, and $\Delta_{j}$, $K_{j}(j=1,2)$ are related to the linear effects. It has been verified that (4) is completely integrable for the case $c_{1}=c_{2}$, $\alpha=\beta=\gamma$ in [6]. Thus, we consider the complete integrable CNLS equations as

$$
\begin{gathered}
i u_{x}+c_{1} u_{t t}+2 \alpha(|u|+|v|) u+\Delta_{+} u+K_{+} v=0, \\
i v_{x}+c_{1} v_{t t}+2 \alpha(|u|+|v|) v+\Delta_{-} u+K_{-} v=0,
\end{gathered}
$$

with $\Delta_{ \pm}=\Delta_{1} \pm \Delta_{2}, K_{ \pm}=K_{1} \pm i K_{2}$, where $c_{1}$ is the group velocity coefficient, $\alpha$ is the parameter related to nonlinear effects, and $\Delta_{ \pm}, K_{ \pm}$are related to linear effects. Although the investigations into the CNLS equations have been conducted via the different methods including Darboux transform [17], the Hirota method [31, 32], extended Jacobi's elliptic function method [18], Painlevés analysis [14], and Bäcklund transformation [33-36] and many soliton solutions have been derived including one-soliton solutions, there are still several points to continue to explore as follows:

(1) Analysis of the CNLS equations and extraction of the bilinear system based on the Hirota method.

(2) Seeking breather solutions and three- (two-) soliton solutions and describing the propagation characters through the numerical simulations.

(3) Analysing the propagation characteristics of collision between multisoliton solutions and breather solutions with different values of parameters.

Thus, we will seek multisolitons, breathers, and collisions between several solitons, respectively, via the Hirota method introduced in [37] and describing the propagation characteristics with figures in the next section. In addition, the conclusion will be given in Section 3, which illustrates the transmission characteristics of the exact solutions contained in the CNLS equations synthetically.

\section{Bilinear Forms and Soliton Solutions}

The Hirota method is a direct analytic tool to acquire the soliton solutions which has been applied to many nonlinear evolution equations (NLEEs) [37-39]. Once the bilinear form of the NLEE was given, multisoliton solutions can be derived through the truncated formal perturbation expansion at different levels [40]. In this section, we will apply the method to construct $\mathrm{N}$-soliton solutions for (5).

Introducing the dependent variable transformation

$$
\begin{aligned}
& u=\frac{g}{f}, \\
& v=\frac{h}{f},
\end{aligned}
$$

into (5), the bilinear form can be derived as follows:

$$
\begin{array}{r}
\left(i D_{x}+c_{1} D_{t}^{2}+\Delta_{+}-i \sqrt{K_{+} \Delta_{-}}\right)(g \cdot f)=0, \\
\left(i D_{x}+c_{1} D_{t}^{2}+K_{-}-i \sqrt{K_{+} \Delta_{-}}\right)(h \cdot f)=0, \\
D_{t}^{2}(f \cdot f)-2 \frac{\alpha}{c_{1}}\left(|g|^{2}+|h|^{2}\right)=0,
\end{array}
$$

where $g, h$ are all complex differential functions to be determined, $f$ is a real one, and the bilinear operators $D_{x}$ and $D_{t}^{2}$ are defined by

$$
\begin{aligned}
& D_{x}^{n} D_{t}^{m}(f \cdot g)=\left(\frac{\partial}{\partial x}-\frac{\partial}{\partial x^{\prime}}\right)^{n}\left(\frac{\partial}{\partial t}-\frac{\partial}{\partial t^{\prime}}\right)^{m} \\
& \quad \times\left. f(x, t) g\left(x^{\prime}, t^{\prime}\right)\right|_{t^{\prime}=t, x^{\prime}=x},
\end{aligned}
$$

where $m$ and $n$ are both positive integers.

Next, we will analyse (7) through expanding $g, h$, and $f$ with the parameter $\varepsilon$, obtain the soliton solutions by symbolic computation, and analyse the propagation characters based on numerical simulation.

2.1. One-Soliton Solutions for (5). In this case, we truncate the perturbation expansion of $g, h$, and $f$ in relation to an expansion parameter $\varepsilon$ as follows:

$$
\begin{aligned}
& g=\varepsilon g_{1}, \\
& h=\varepsilon h_{1}, \\
& f=1+\varepsilon^{2} f_{1} .
\end{aligned}
$$

Substituting (9) into (7), setting $\varepsilon=1$, we solve the bilinear system recursively and obtain the analytical onesoliton solution as 


$$
\begin{aligned}
& u=\frac{e^{t \omega_{1}+x\left(\sqrt{\Delta_{-} K_{+}}+i\left(\Delta_{+}+c_{1} \omega_{1}^{2}\right)\right)}}{1+\left(X / c_{1}\right)\left(e^{t\left(\omega_{3}+\bar{\omega}_{3}\right)+x \sqrt{\Delta_{-} K_{+}}+x\left(\sqrt{\bar{\Delta}_{-} \bar{K}_{+}}+i\left(\Delta_{+}-\bar{\Delta}_{+}+c_{1}\left(\omega_{1}^{2}-\bar{\omega}_{1}^{2}\right)\right)\right)} /\left(\omega_{3}+\bar{\omega}_{3}\right)^{2}\right)}, \\
& v=\frac{-i \sqrt{\Delta_{-} / K_{+}} e^{t \omega_{1}+x\left(\sqrt{\Delta_{-} K_{+}}+i\left(\Delta_{+}+c_{1} \omega_{1}^{2}\right)\right)}}{1+\left(X / c_{1}\right)\left(e^{t\left(\omega_{3}+\bar{\omega}_{3}\right)+x \sqrt{\Delta_{-} K_{+}}+x\left(\sqrt{\bar{\Delta}_{-} \bar{K}_{+}}+i\left(\Delta_{+}-\bar{\Delta}_{+}+c_{1}\left(\omega_{1}^{2}-\bar{\omega}_{1}^{2}\right)\right)\right)} /\left(\omega_{3}+\bar{\omega}_{3}\right)^{2}\right)},
\end{aligned}
$$

with

$$
\begin{aligned}
\theta_{1}= & k_{1} x+\omega_{1} t, \\
\theta_{3}= & k_{3} x+\omega_{3} t, \\
X= & \alpha\left(1+\sqrt{\frac{\Delta_{-}}{K_{+}}} \sqrt{\frac{\bar{\Delta}_{-}}{\bar{K}_{+}}}\right), \\
K_{-}= & \Delta_{+}, \\
k_{3}= & \sqrt{\Delta_{-} K_{+}}+\sqrt{\bar{\Delta}_{-} \bar{K}_{+}} \\
& +i\left(\Delta_{+}-\bar{\Delta}_{+}+c_{1}\left(\omega_{1}^{2}-\bar{\omega}_{1}^{2}\right)\right), \\
k_{1}= & \sqrt{\Delta_{-} K_{+}}+i\left(\Delta_{+}+c_{1} \omega_{1}^{2}\right), \\
g_{1}= & \exp \left(\theta_{1}\right), \\
h_{1}= & -i \sqrt{\frac{\Delta_{-}}{K_{+}}} \exp \left(\theta_{1}\right), \\
f_{1}= & \delta \exp \left(\theta_{3}+\bar{\theta}_{3}\right), \\
\delta= & \frac{X}{c_{1}\left(\omega_{3}+\bar{\omega}_{3}\right)^{2}},
\end{aligned}
$$

where $c_{1}, \delta$, and $\Delta_{ \pm}$are real constants and $K_{ \pm}$are both complex ones.

By choosing different parameters, soliton solutions can be derived. Figure 1(a) displays the propagation characteristics of the bright soliton solution. It can be seen in Figure 1(b) that presents boundedness in amplitude and periodicity in the process of propagation, which is similar to the one in Figure 1(c) with the modification of the parameter $\omega_{3}$.

2.2. Two-Soliton Solutions for (5). Similar to the procedure in Section 2.1, we truncate the perturbation expansion of $g, h$, and $f$ with respect to the parameter $\varepsilon$ as

$$
\begin{aligned}
& g=\varepsilon g_{1}+\varepsilon^{3} g_{3}, \\
& h=\varepsilon h_{1}+\varepsilon^{3} h_{3}, \\
& f=1+\varepsilon^{2} f_{2}+\varepsilon^{4} f_{4} .
\end{aligned}
$$

Substituting (12) into (7), solving the bilinear system recursively, and setting $\varepsilon=1, \varphi_{1}=0$, and $\varphi_{2}=0$, the analytical two-soliton solutions can be derived as follows:

$u$

$$
\begin{gathered}
=\frac{e^{p_{1}}+e^{p_{2}}+\left(\alpha \Lambda / c_{1}\right)\left(e^{p_{1}+r_{2}+\bar{r}_{1}} /\left(\omega_{1}+\bar{\omega}_{1}\right)^{2}\left(\omega_{2}+\bar{\omega}_{1}\right)^{2}+e^{p_{1}+r_{2}+\bar{r}_{2}} /\left(\omega_{2}+\bar{\omega}_{2}\right)^{2}\left(\omega_{1}+\bar{\omega}_{2}\right)^{2}\right)}{1+\left(\alpha \Lambda / c_{1}\right)\left(e^{r_{1}+\bar{r}_{1}} /\left(\omega_{1}+\bar{\omega}_{1}\right)^{2}+e^{r_{2}+\bar{r}_{1}} /\left(\omega_{2}+\bar{\omega}_{1}\right)^{2}+e^{r_{1}+\bar{r}_{2}} /\left(\omega_{1}+\bar{\omega}_{2}\right)^{2}+e^{r_{2}+\bar{r}_{2}} /\left(\omega_{2}+\bar{\omega}_{2}\right)^{2}\right)+\left(\alpha^{2} \Gamma / \Phi\right) e^{r_{1}+\bar{r}_{1}+r_{2}+\bar{r}_{2}}}, \\
v=\frac{i \sqrt{\Delta_{-} / K_{+}}\left(e^{p_{1}}+e^{p_{2}}+\left(\alpha \Lambda / c_{1}\right)\left(e^{p_{1}+r_{2}+\bar{r}_{1}} /\left(\omega_{1}+\bar{\omega}_{1}\right)^{2}\left(\omega_{2}+\bar{\omega}_{1}\right)^{2}+e^{p_{1}+r_{2}+\bar{r}_{2}} /\left(\omega_{2}+\bar{\omega}_{2}\right)^{2}\left(\omega_{1}+\bar{\omega}_{2}\right)^{2}\right)\right)}{1+\left(\alpha \Lambda / c_{1}\right)\left(e^{r_{1}+\bar{r}_{1}} /\left(\omega_{1}+\bar{\omega}_{1}\right)^{2}+e^{r_{2}+\bar{r}_{1}} /\left(\omega_{2}+\bar{\omega}_{1}\right)^{2}+e^{r_{1}+\bar{r}_{2}} /\left(\omega_{1}+\bar{\omega}_{2}\right)^{2}+e^{r_{2}+\bar{r}_{2}} /\left(\omega_{2}+\bar{\omega}_{2}\right)^{2}\right)+\left(\alpha^{2} \Gamma / \Phi\right) e^{r_{1}+\bar{r}_{1}+r_{2}+\bar{r}_{2}}},
\end{gathered}
$$

with

$$
\begin{aligned}
\theta_{1} & =k_{1} x+\omega_{1} t+\varphi_{1}, \\
\theta_{2} & =k_{2} x+\omega_{2} t+\varphi_{2}, \\
f_{4} & =\sigma_{5} \exp \left(\theta_{1}+\bar{\theta}_{1}+\theta_{2}+\bar{\theta}_{2}\right), \\
f_{2} & =\sigma_{1} \exp \left(\theta_{1}+\bar{\theta}_{1}\right)+\sigma_{2} \exp \left(\theta_{2}+\bar{\theta}_{2}\right)+\sigma_{3} \\
& \cdot \exp \left(\theta_{1}+\bar{\theta}_{2}\right)+\sigma_{4} \exp \left(\theta_{2}+\bar{\theta}_{1}\right),
\end{aligned}
$$

$$
\begin{aligned}
& g_{1}=\exp \left(\theta_{1}\right)+\exp \left(\theta_{2}\right), \\
& g_{3}=\eta_{1} \exp \left(\theta_{1}+\bar{\theta}_{1}+\theta_{2}\right)+\eta_{2} \exp \left(\theta_{2}+\bar{\theta}_{2}+\theta_{1}\right), \\
& k_{1}=\sqrt{\Delta_{-} K_{+}}+i\left(\Delta_{+}+c_{1} \omega_{1}^{2}\right), \\
& k_{2}=\sqrt{\Delta_{-} K_{+}}+i\left(\Delta_{+}+c_{1} \omega_{2}^{2}\right), \\
& \bar{\Delta}_{+}=\Delta_{+}-i\left(\sqrt{\Delta_{-} K_{+}}+\sqrt{\overline{\Delta_{-} \bar{K}_{+}}}\right),
\end{aligned}
$$




$$
\begin{aligned}
\Lambda & =\left(1+\sqrt{\frac{\Delta_{-}}{K_{+}}} \sqrt{\frac{\bar{\Delta}_{-}}{\bar{K}_{+}}}\right)\left(\omega_{1}-\omega_{2}\right)^{2} \\
p_{1} & =t \omega_{1}+x\left(\sqrt{\Delta_{-} K_{+}}+i\left(\Delta_{+}+c_{1} \omega_{1}^{2}\right)\right) \\
p_{2} & =t \omega_{2}+x\left(\sqrt{\Delta_{-} K_{+}}+i\left(\Delta_{+}+c_{1} \omega_{2}^{2}\right)\right) \\
r_{1} & =t \omega_{1}+i x c_{1} \omega_{1}^{2}, \\
r_{2} & =t \omega_{2}+i x c_{1} \omega_{2}^{2} t \omega_{2}, \\
\bar{r}_{1} & =t \bar{\omega}_{1}-i x c_{1} \bar{\omega}_{1}^{2}, \\
\bar{r}_{2} & =t \bar{\omega}_{2}-i x c_{1} \bar{\omega}_{2}^{2}, \\
K_{-} & =\Delta_{+}, \\
\Gamma & =\left(\Delta_{-} \bar{\Delta}_{-}+K_{+} \bar{K}_{+}\left(\frac{2 \Lambda}{\left(\omega_{1}-\omega_{2}\right)^{2}}-1\right)\right)\left(\omega_{1}-\omega_{2}\right)^{2} \\
& \cdot\left(\bar{\omega}_{1}-\bar{\omega}_{2}\right)^{2}, \\
& =c_{1}^{2} K_{+} \bar{K}_{+}\left(\omega_{1}+\bar{\omega}_{2}\right)^{2}, \\
& \\
&
\end{aligned}
$$

where $c_{1}, K_{ \pm}$, and $\Delta_{ \pm}$are the same as those in (10); other parameters can be seen in Appendix A. Obviously, Figure 2(a) describes the propagation characteristics of the two-soliton solution. Changing the parameters $\alpha, K_{+}$, and $\omega_{2}$, Figure 2(b) can be described certainly, which reveals the propagation process with the higher impact velocity than that in Figure 2(a) due to decreasing of the values of $\alpha, K_{+}$, and $\omega_{2}$; and setting the values of $\omega_{1}, \omega_{2}$, and $\omega_{3}$ to real constants, soliton solutions are derived in Figures 2(c) and 2(d), which describe the bright soliton and the breather propagate in parallel. The relationship in the space coordinate between the bright soliton and the breather also varies as changing the value of $\omega_{2}$.

2.3. Three-Soliton Solutions for (5). By treating the truncated perturbation expansions of $g, h$, and $f$ as

$$
\begin{aligned}
& g=\varepsilon g_{1}+\varepsilon^{3} g_{3}+\varepsilon^{5} g_{5}, \\
& h=\varepsilon h_{1}+\varepsilon^{3} h_{3}+\varepsilon^{5} h_{5}, \\
& f=1+\varepsilon^{2} f_{2}+\varepsilon^{4} f_{4}+\varepsilon^{6} f_{6},
\end{aligned}
$$

we give the three-soliton solutions for (5) as follows:

$$
\begin{aligned}
& u=\frac{g_{1}+g_{3}+g_{5}}{1+f_{2}+f_{4}+f_{6}}, \\
& v=\frac{h_{1}+h_{3}+h_{5}}{1+f_{2}+f_{4}+f_{6}},
\end{aligned}
$$

with

$$
\begin{aligned}
& \theta_{1}=k_{1} x+\omega_{1} t+\varphi_{1}, \\
& \theta_{2}=k_{2} x+\omega_{2} t+\varphi_{2} \text {, } \\
& \theta_{3}=k_{3} x+\omega_{3} t+\varphi_{3} \text {, } \\
& g_{1}=\exp \left(\theta_{1}\right)+\exp \left(\theta_{2}\right)+\exp \left(\theta_{3}\right) \text {, } \\
& f_{6}=\Omega \exp \left(\theta_{1}+\theta_{2}+\theta_{3}+\bar{\theta}_{1}+\bar{\theta}_{2}+\bar{\theta}_{3}\right) \text {, } \\
& g_{3}=\eta_{1} \exp \left(\theta_{1}+\bar{\theta}_{1}+\theta_{2}\right)+\eta_{2} \exp \left(\theta_{1}+\bar{\theta}_{2}+\theta_{2}\right) \\
& +\eta_{3} \exp \left(\theta_{1}+\bar{\theta}_{3}+\theta_{2}\right) \\
& +\eta_{4} \exp \left(\theta_{1}+\bar{\theta}_{1}+\theta_{3}\right) \\
& +\eta_{5} \exp \left(\theta_{1}+\bar{\theta}_{2}+\theta_{3}\right) \\
& +\eta_{6} \exp \left(\theta_{3}+\bar{\theta}_{1}+\theta_{2}\right) \\
& +\eta_{7} \exp \left(\theta_{3}+\bar{\theta}_{2}+\theta_{2}\right) \\
& +\eta_{8} \exp \left(\theta_{1}+\bar{\theta}_{3}+\theta_{3}\right) \\
& +\eta_{9} \exp \left(\theta_{3}+\bar{\theta}_{3}+\theta_{2}\right) \text {, } \\
& g_{5}=\chi_{1} \exp \left(\theta_{1}+\theta_{2}+\theta_{3}+\bar{\theta}_{1}+\bar{\theta}_{2}\right) \\
& +\chi_{2} \exp \left(\theta_{1}+\theta_{2}+\theta_{3}+\bar{\theta}_{1}+\bar{\theta}_{3}\right) \\
& +\chi_{3} \exp \left(\theta_{1}+\theta_{2}+\theta_{3}+\bar{\theta}_{3}+\bar{\theta}_{2}\right) \text {, } \\
& f_{2}=\sigma_{1} \exp \left(\theta_{1}+\bar{\theta}_{1}\right)+\sigma_{2} \exp \left(\theta_{2}+\bar{\theta}_{2}\right) \\
& +\sigma_{3} \exp \left(\theta_{1}+\bar{\theta}_{2}\right)+\sigma_{4} \exp \left(\theta_{2}+\bar{\theta}_{1}\right) \\
& +\sigma_{5} \exp \left(\theta_{3}+\bar{\theta}_{3}\right)+\sigma_{6} \exp \left(\theta_{3}+\bar{\theta}_{1}\right) \\
& +\sigma_{7} \exp \left(\theta_{3}+\bar{\theta}_{2}\right)+\sigma_{8} \exp \left(\theta_{2}+\bar{\theta}_{3}\right) \\
& +\sigma_{9} \exp \left(\theta_{1}+\bar{\theta}_{3}\right) \\
& f_{4}=\rho_{1} \exp \left(\theta_{1}+\theta_{2}+\bar{\theta}_{2}+\bar{\theta}_{1}\right) \\
& +\rho_{2} \exp \left(\theta_{1}+\theta_{3}+\bar{\theta}_{3}+\bar{\theta}_{1}\right) \\
& +\rho_{3} \exp \left(\theta_{3}+\theta_{2}+\bar{\theta}_{2}+\bar{\theta}_{3}\right) \\
& +\rho_{4} \exp \left(\theta_{1}+\theta_{2}+\bar{\theta}_{3}+\bar{\theta}_{1}\right) \\
& +\rho_{5} \exp \left(\theta_{1}+\theta_{2}+\bar{\theta}_{2}+\bar{\theta}_{3}\right) \\
& +\rho_{6} \exp \left(\theta_{1}+\theta_{3}+\bar{\theta}_{2}+\bar{\theta}_{1}\right) \\
& +\rho_{7} \exp \left(\theta_{1}+\theta_{3}+\bar{\theta}_{2}+\bar{\theta}_{3}\right) \\
& +\rho_{8} \exp \left(\theta_{3}+\theta_{2}+\bar{\theta}_{2}+\bar{\theta}_{1}\right) \\
& +\rho_{9} \exp \left(\theta_{3}+\theta_{2}+\bar{\theta}_{3}+\bar{\theta}_{1}\right),
\end{aligned}
$$



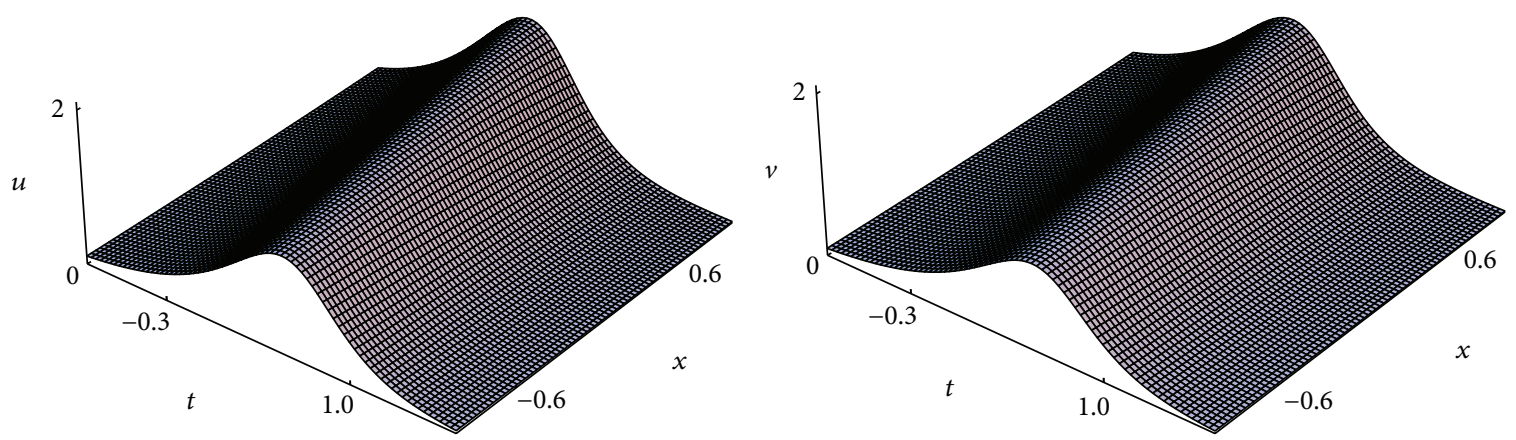

(a)
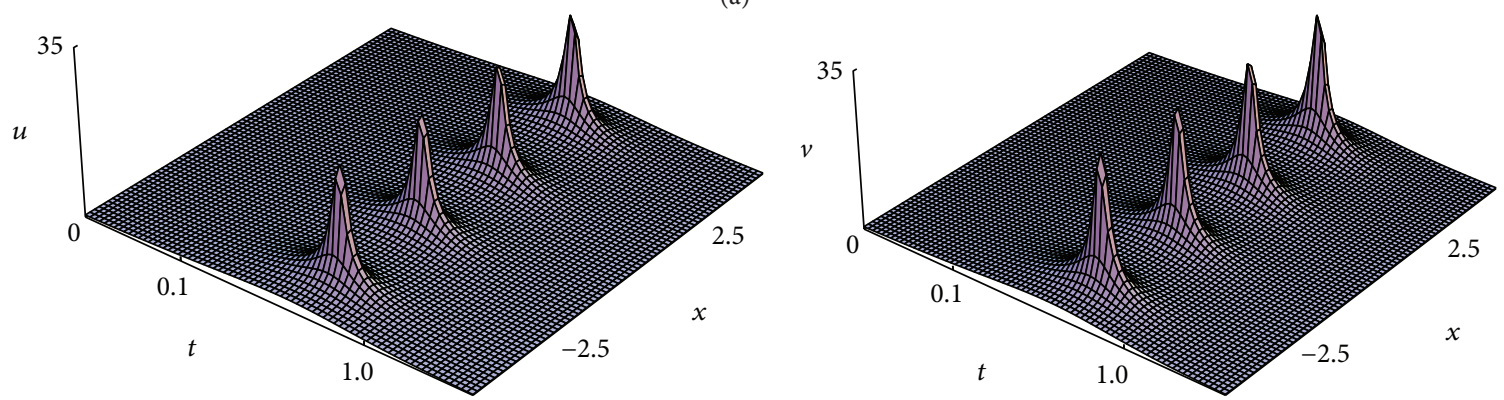

(b)
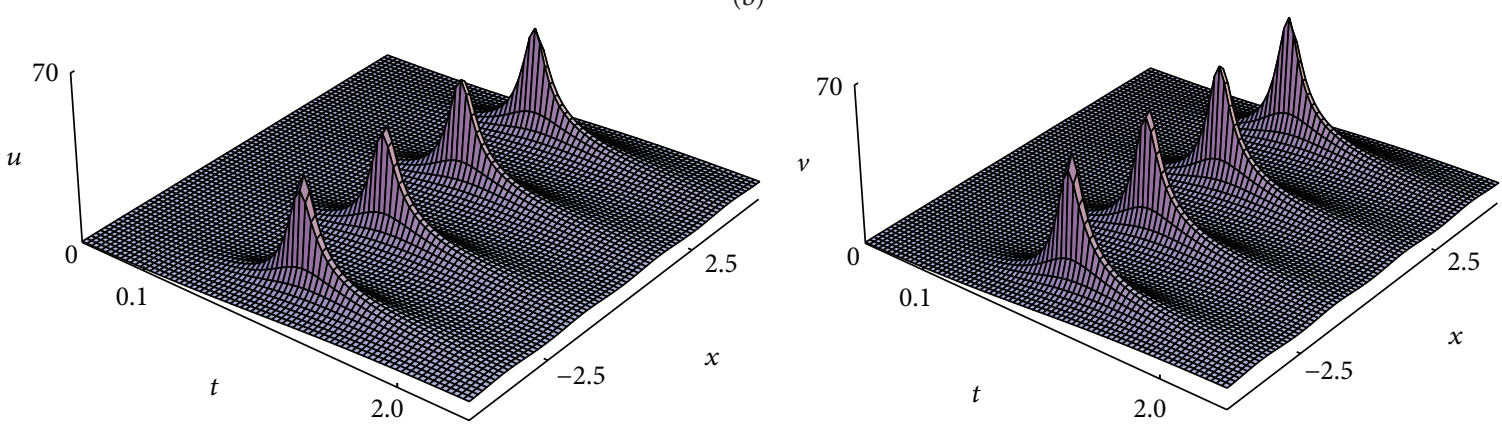

(c)

Figure 1: Evolution of the solutions of (10). (a) Parameters are $c_{1}=1, \alpha=1, K_{+}=1-i, \Delta_{-}=-1, \Delta_{+}=1, \omega_{1}=2$, and $\omega_{3}=3$. (b) With the same parameters as those in (a) except for $c_{1}=-1, K_{+}=-1, \Delta_{-}=1.5$, and $\Delta_{+}=2$. (c) With the same parameters as those in (b) except for $\omega_{3}=1$.

where $\varphi_{1}, \varphi_{2}$, and $\varphi_{3}$ are the same as those in (13); other parameters can be seen in Appendix B. According to (16), with the parameters $\omega_{1}, \omega_{2}$, and $\omega_{3}$ being complex numbers, we can obtain a precise three-bright-soliton in Figure 3(a); with the parameters $\omega_{1}, \omega_{2}$, and $\omega_{3}$ being real ones, we can derive a precise three-breather in Figure 3(b). Decreasing the value of $K_{+}, \alpha$, and $\omega_{3}$ while keeping other parameters as those in Figure 3(b), we obtain the colliding bright soliton with breather in Figure 3(c). Keeping the parameters the same as those in Figure 3(a), except for $\omega_{1}=2$, Figure 4(a) is derived. In contrast to the case in Figure 3(a), if setting $\omega_{2}=-2$, we can obtain the opposite propagation process on $x$ coordinate in Figure 4(b).

\section{Conclusions}

In this paper, our main focus is on (5) with the linear effects. Through the constructed Hirota system, we obtain one-soliton solutions in Figure 1, two-soliton solutions in Figure 2, and three-soliton solutions in Figures 3 and 4 via symbolic computation, which describe the propagation characters of solitons with different values of parameters. Figure 1 describes one bright soliton in (a) and the bound soliton in (b) and (c) as the parameter $\omega_{3}$ changes. Obviously, it shows in Figure 2 that two-bright-soliton turns to the parallel propagation between a bright soliton and a breather with change of the values of $\Delta_{+}, \omega_{1}$, and $\omega_{3}$. Choosing suitable values of $\omega_{1}$ and $\omega_{3}$ in Figure 2(a), the three-brightsoliton is derived in Figure 3(a). In particular, we seek out the three-breathers in Figure 3(b) with the real constants of $\omega_{1}, \omega_{2}$, and $\omega_{3}$. However, when one of $\omega_{1}, \omega_{2}$, and $\omega_{3}$ is a complex one, we can receive the propagation process of the evolution between the bright soliton and the breather in Figure 4. Using all the above, via setting the key parameters to different values, we obtain optical multisoliton solutions with distinctive propagation characteristics. 

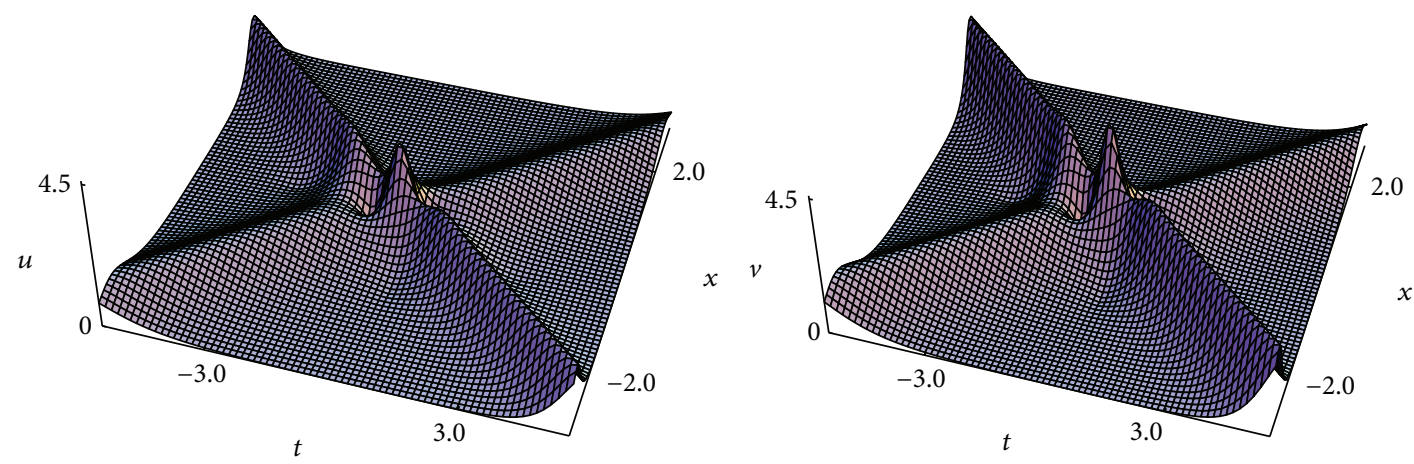

(a)
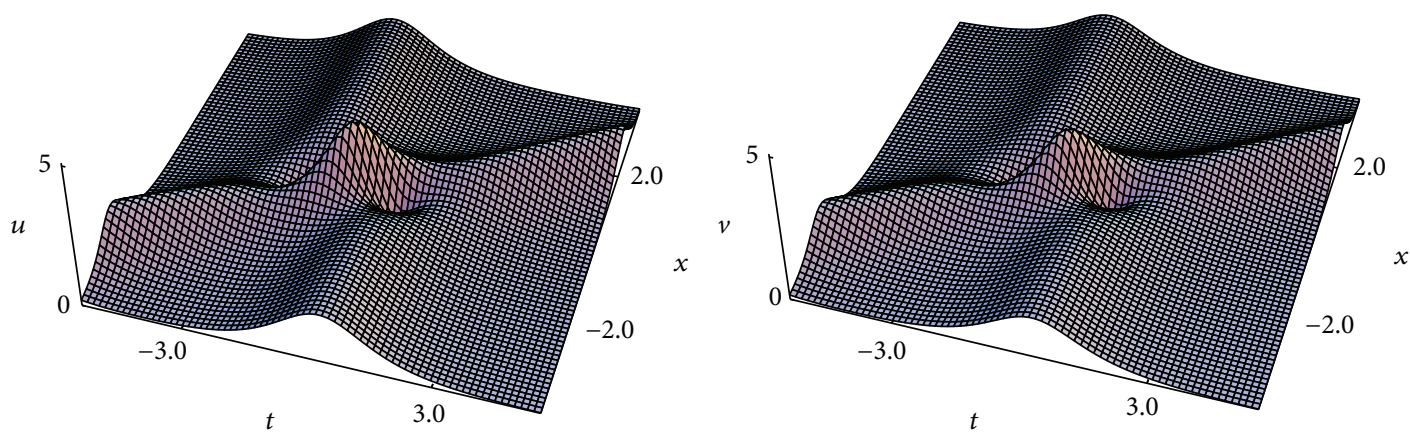

(b)
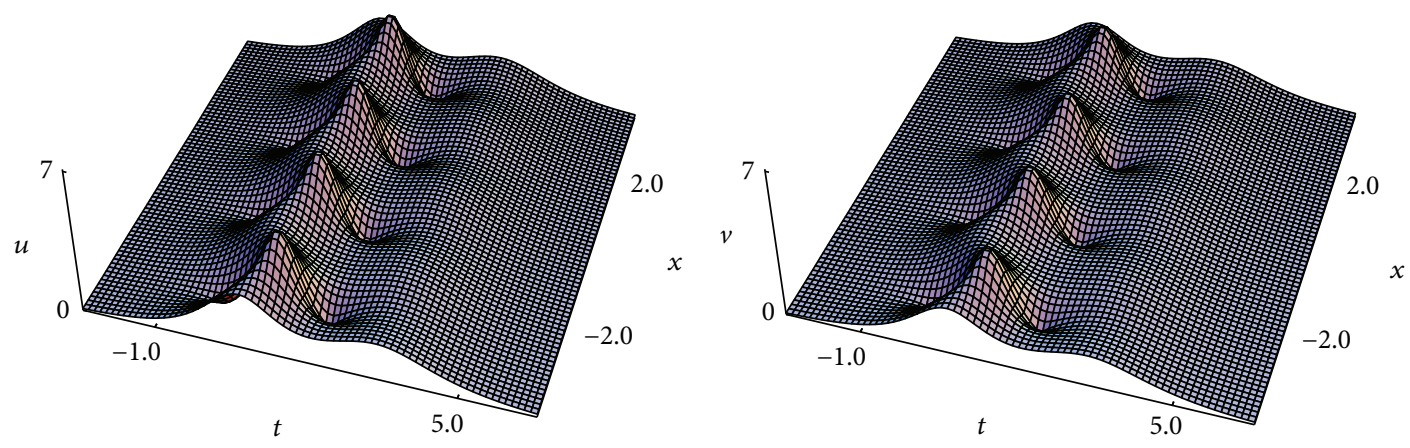

(c)
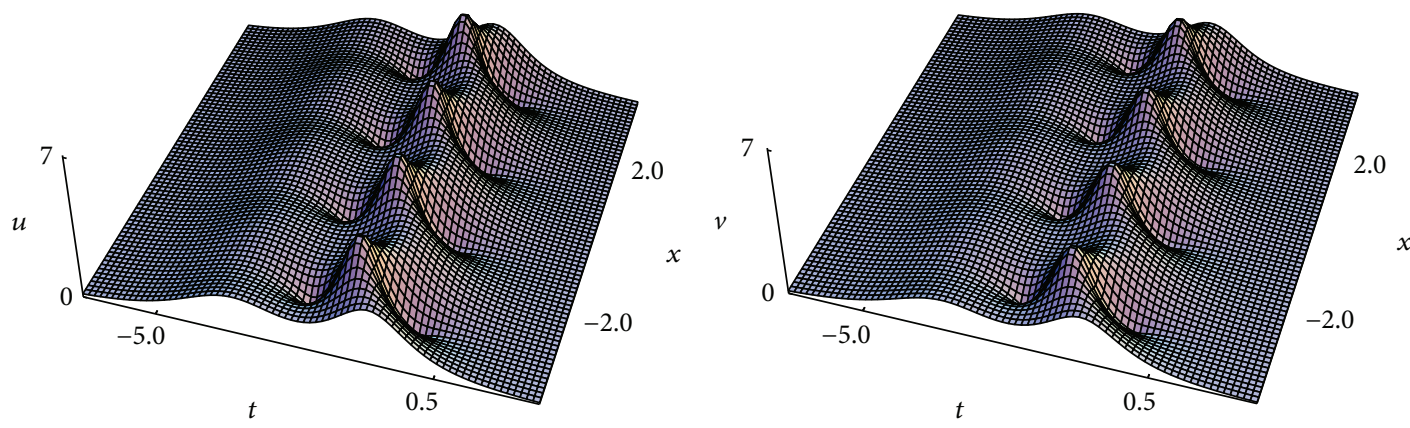

(d)

Figure 2: Evolution of the solutions of (13). (a) Parameters are $c_{1}=-1, \alpha=1, K_{+}=1, \Delta_{-}=-2, \Delta_{+}=2, \omega_{1}=1-i$, and $\omega_{3}=-2+i$. (b) Parameters are $c_{1}=-1, \alpha=-1, K_{+}=-3, \Delta_{-}=2, \Delta_{+}=3, \omega_{1}=1$, and $\omega_{3}=2$. (c) With the same parameters as those in (b) except for $\Delta_{+}=-3, \omega_{1}=-1$, and $\omega_{3}=-2$. (d) With the same parameters as those in (b) except for $\Delta_{+}=-1, \omega_{1}=1-i$, and $\omega_{3}=-1$. 

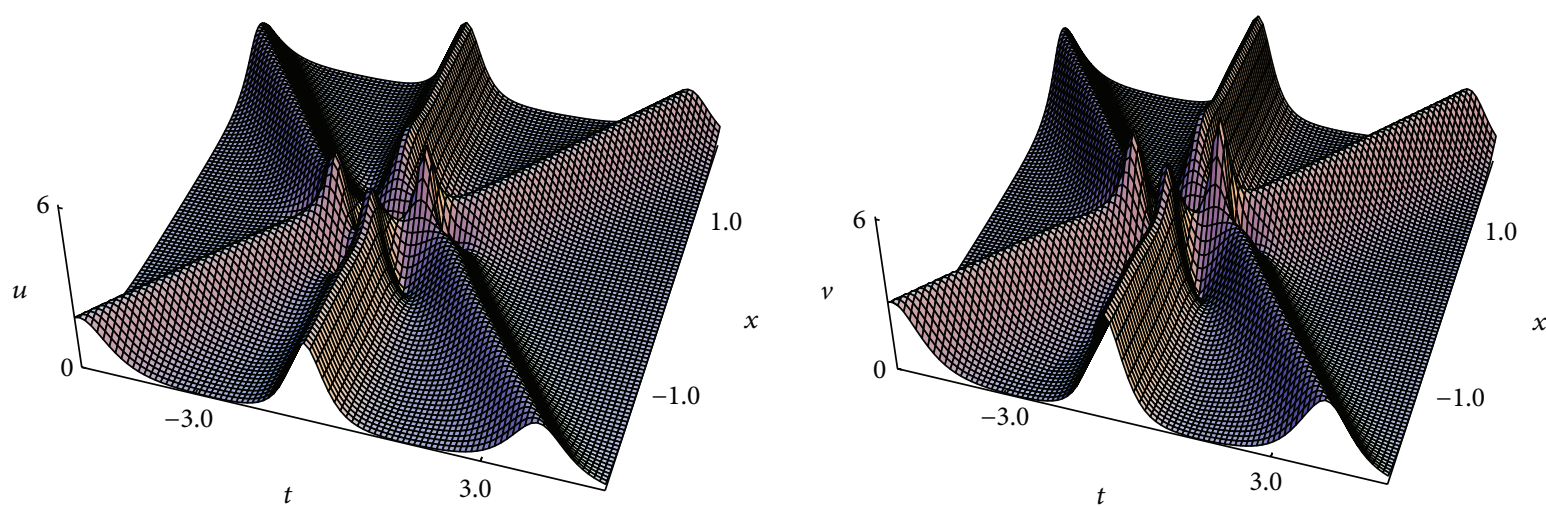

(a)
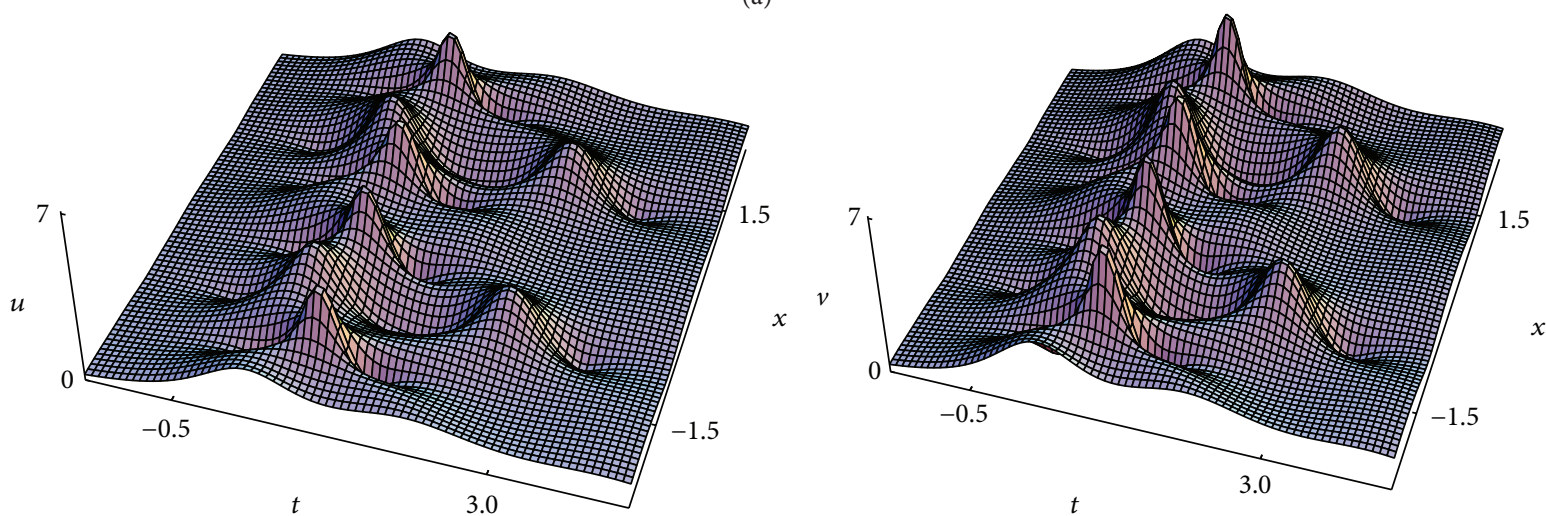

(b)
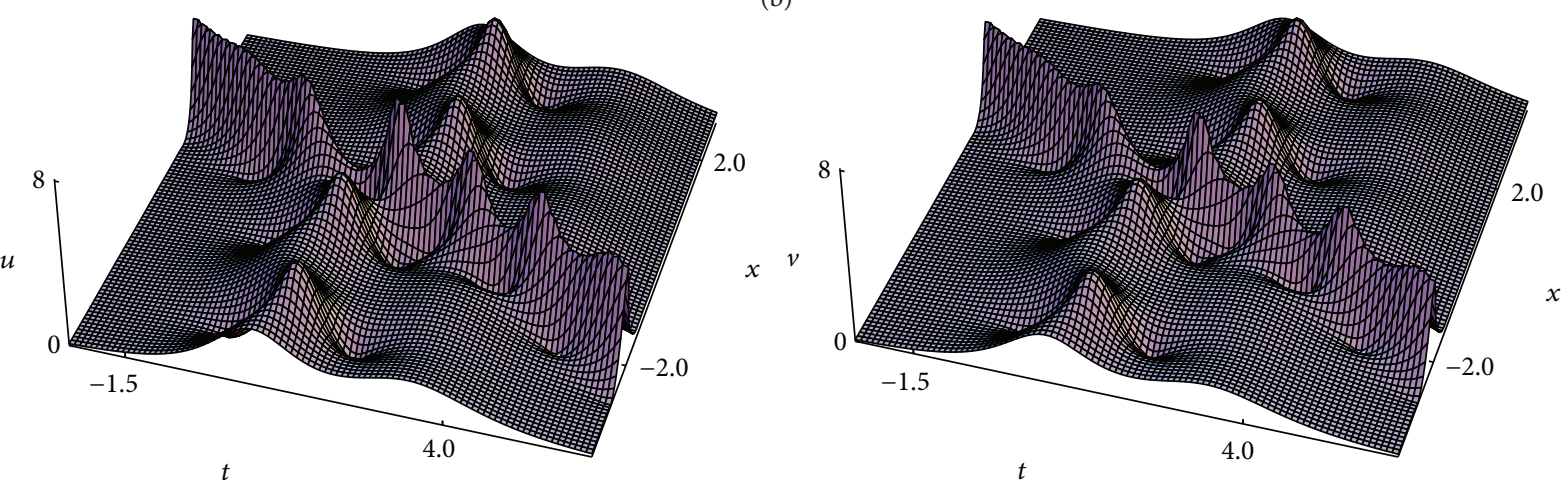

(c)

FIgURE 3: Evolution of the solutions of (16). (a) Parameters are $c_{1}=-1, \alpha=1, K_{+}=1, \Delta_{-}=-2, \Delta_{+}=2, \omega_{1}=2-i, \omega_{2}=-2+i$, and $\omega_{3}=-3$. (b) With the same parameters as those in (a) except for $\omega_{1}=1, \omega_{2}=2$, and $\omega_{3}=3$. (c) With the same parameters as those in (b) except for $\alpha=-1, K_{+}=-3, \Delta_{-}=2$, and $\omega_{3}=3+i$.

\section{Appendix}

\section{A. The Parameters of the Two-Soliton Solutions for (5)}

Corresponding parameters in solution equations (13) are expressed as

$$
\begin{aligned}
\Gamma & =\left(\Delta_{-} \bar{\Delta}_{-}+K_{+} \bar{K}_{+}(2 \Lambda-1)\right), \\
\Lambda & =\left(1+\sqrt{\frac{\Delta_{-}}{K_{+}}} \sqrt{\frac{\bar{\Delta}_{-}}{\bar{K}_{+}}}\right),
\end{aligned}
$$

$$
\begin{aligned}
\sigma_{1} & =\frac{\alpha \Lambda}{c_{1}\left(\omega_{1}+\bar{\omega}_{1}\right)^{2}}, \\
\sigma_{2} & =\frac{\alpha \Lambda}{c_{1}\left(\omega_{2}+\bar{\omega}_{2}\right)^{2}}, \\
\sigma_{3} & =\frac{\alpha \Lambda}{c_{1}\left(\omega_{1}+\bar{\omega}_{2}\right)^{2}}, \\
\sigma_{4} & =\frac{\alpha \Lambda}{c_{1}\left(\omega_{2}+\bar{\omega}_{1}\right)^{2}}, \\
\eta_{1} & =\frac{\alpha \Lambda\left(\omega_{1}-\omega_{2}\right)^{2}}{c_{1}\left(\omega_{1}+\bar{\omega}_{1}\right)^{2}\left(\bar{\omega}_{1}+\omega_{2}\right)^{2}},
\end{aligned}
$$




$$
\begin{aligned}
& \sigma_{5} \\
& =\frac{\alpha^{2} \Gamma\left(\omega_{1}-\omega_{2}\right)^{2}\left(\bar{\omega}_{1}-\bar{\omega}_{2}\right)^{2}}{K_{+} \bar{K}_{+} c_{1}^{2}\left(\omega_{2}+\bar{\omega}_{2}\right)^{2}\left(\omega_{1}+\bar{\omega}_{1}\right)^{2}\left(\omega_{1}+\bar{\omega}_{2}\right)^{2}\left(\omega_{2}+\bar{\omega}_{1}\right)^{2}}, \\
& \eta_{2}=\frac{\alpha \Lambda\left(\omega_{1}-\omega_{2}\right)^{2}}{c_{1}\left(\omega_{1}+\bar{\omega}_{2}\right)^{2}\left(\bar{\omega}_{2}+\omega_{2}\right)^{2}},
\end{aligned}
$$

\section{B. The Parameters of the Three-Soliton Solutions for (5)}

With the same value of $\Gamma$ and $\Lambda$ as those in Appendix A, corresponding parameters in solution equations (16) are expressed as

where $c_{1}$ and $\alpha$ are real constants.

$$
\begin{aligned}
& \sigma_{1}=\frac{\alpha \Lambda}{c_{1}\left(\omega_{1}+\bar{\omega}_{1}\right)^{2}}, \\
& \sigma_{2}=\frac{\alpha \Lambda}{c_{1}\left(\omega_{2}+\bar{\omega}_{2}\right)^{2}} \text {, } \\
& \sigma_{3}=\frac{\alpha \Lambda}{c_{1}\left(\omega_{1}+\bar{\omega}_{2}\right)^{2}}, \\
& \sigma_{4}=\frac{\alpha \Lambda}{c_{1}\left(\omega_{2}+\bar{\omega}_{1}\right)^{2}}, \\
& \sigma_{5}=\frac{\alpha \Lambda}{c_{1}\left(\omega_{3}+\bar{\omega}_{3}\right)^{2}}, \\
& \sigma_{6}=\frac{\alpha \Lambda}{c_{1}\left(\omega_{3}+\bar{\omega}_{1}\right)^{2}} \text {, } \\
& \sigma_{7}=\frac{\alpha \Lambda}{c_{1}\left(\omega_{3}+\bar{\omega}_{2}\right)^{2}} \text {, } \\
& \sigma_{8}=\frac{\alpha \Lambda}{c_{1}\left(\omega_{2}+\bar{\omega}_{3}\right)^{2}} \text {, } \\
& \sigma_{9}=\frac{\alpha \Lambda}{c_{1}\left(\omega_{1}+\bar{\omega}_{3}\right)^{2}} \text {, } \\
& \chi_{1}=\frac{\alpha^{2} \Gamma\left(\omega_{1}-\omega_{2}\right)^{2}\left(\omega_{1}-\omega_{3}\right)^{2}\left(\omega_{2}-\omega_{3}\right)^{2}\left(\bar{\omega}_{1}-\bar{\omega}_{2}\right)^{2}}{c_{1}^{2} K_{+} \bar{K}_{+}\left(\omega_{1}+\bar{\omega}_{1}\right)^{2}\left(\omega_{2}+\bar{\omega}_{1}\right)^{2}\left(\omega_{3}+\bar{\omega}_{1}\right)^{2}\left(\omega_{1}+\bar{\omega}_{2}\right)^{2}\left(\omega_{2}+\bar{\omega}_{2}\right)^{2}\left(\omega_{3}+\bar{\omega}_{2}\right)^{2}}, \\
& \chi_{2}=\frac{\alpha^{2} \Gamma\left(\omega_{1}-\omega_{2}\right)^{2}\left(\omega_{1}-\omega_{3}\right)^{2}\left(\omega_{2}-\omega_{3}\right)^{2}\left(\bar{\omega}_{1}-\bar{\omega}_{3}\right)^{2}}{c_{1}^{2} K_{+} \bar{K}_{+}\left(\omega_{1}+\bar{\omega}_{1}\right)^{2}\left(\omega_{2}+\bar{\omega}_{1}\right)^{2}\left(\omega_{3}+\bar{\omega}_{1}\right)^{2}\left(\omega_{1}+\bar{\omega}_{3}\right)^{2}\left(\omega_{2}+\bar{\omega}_{3}\right)^{2}\left(\omega_{3}+\bar{\omega}_{3}\right)^{2}}, \\
& \chi_{3}=\frac{\alpha^{2} \Gamma\left(\omega_{1}-\omega_{2}\right)^{2}\left(\omega_{1}-\omega_{3}\right)^{2}\left(\omega_{2}-\omega_{3}\right)^{2}\left(\bar{\omega}_{2}-\bar{\omega}_{3}\right)^{2}}{c_{1}^{2} K_{+} \bar{K}_{+}\left(\omega_{2}+\bar{\omega}_{2}\right)^{2}\left(\omega_{1}+\bar{\omega}_{2}\right)^{2}\left(\omega_{3}+\bar{\omega}_{2}\right)^{2}\left(\omega_{1}+\bar{\omega}_{3}\right)^{2}\left(\omega_{2}+\bar{\omega}_{3}\right)^{2}\left(\omega_{3}+\bar{\omega}_{3}\right)^{2}} \text {, } \\
& \rho_{1}=\frac{\alpha^{2} \Gamma\left(\omega_{1}-\omega_{2}\right)^{2}\left(\bar{\omega}_{1}-\bar{\omega}_{2}\right)^{2}}{c_{1}^{2} K_{+} \bar{K}_{+}\left(\omega_{1}+\bar{\omega}_{1}\right)^{2}\left(\omega_{2}+\bar{\omega}_{1}\right)^{2}\left(\omega_{1}+\bar{\omega}_{2}\right)^{2}\left(\bar{\omega}_{2}+\omega_{2}\right)^{2}} \text {, } \\
& \eta_{1}=\frac{\alpha \Lambda\left(\omega_{1}-\omega_{2}\right)^{2}}{c_{1}\left(\omega_{1}+\bar{\omega}_{1}\right)^{2}\left(\bar{\omega}_{1}+\omega_{2}\right)^{2}} \\
& \rho_{2}=\frac{\alpha^{2} \Gamma\left(\omega_{1}-\omega_{3}\right)^{2}\left(\bar{\omega}_{1}-\bar{\omega}_{3}\right)^{2}}{c_{1}^{2} K_{+} \bar{K}_{+}\left(\omega_{1}+\bar{\omega}_{1}\right)^{2}\left(\omega_{3}+\bar{\omega}_{1}\right)^{2}\left(\omega_{1}+\bar{\omega}_{3}\right)^{2}\left(\bar{\omega}_{3}+\omega_{3}\right)^{2}}, \\
& \eta_{2}=\frac{\alpha \Lambda\left(\omega_{1}-\omega_{2}\right)^{2}}{c_{1}\left(\omega_{1}+\bar{\omega}_{2}\right)^{2}\left(\bar{\omega}_{2}+\omega_{2}\right)^{2}}
\end{aligned}
$$



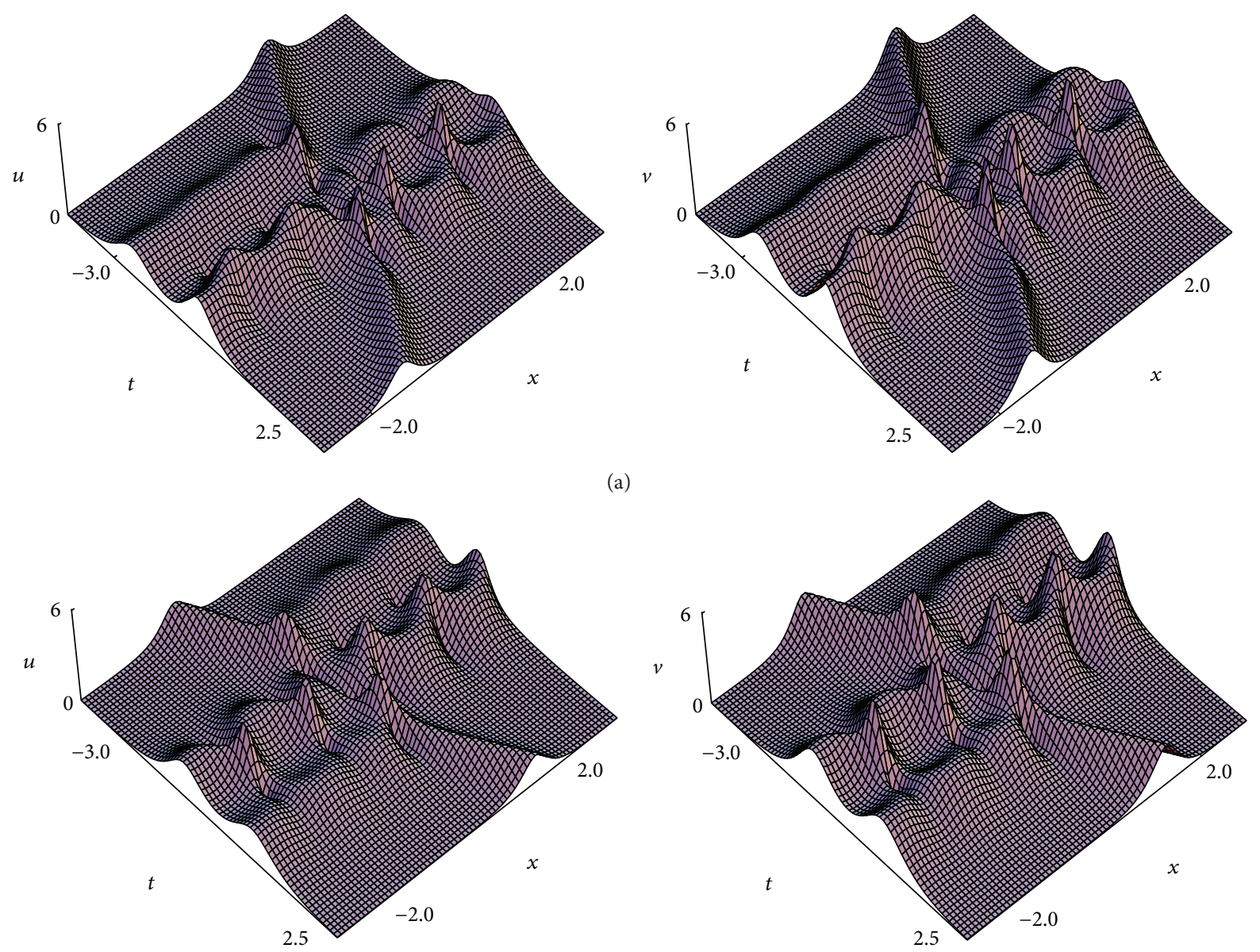

(b)

Figure 4: Evolution of the solutions of (13). (a) With the same parameters as those in Figure 3(a) except for $\omega_{1}=2$. (b) With the same parameters as those in (a) except for $\omega_{2}=-2$.

$$
\begin{aligned}
& \rho_{3}=\frac{\alpha^{2} \Gamma\left(\omega_{2}-\omega_{3}\right)^{2}\left(\bar{\omega}_{2}-\bar{\omega}_{3}\right)^{2}}{c_{1}^{2} K_{+} \bar{K}_{+}\left(\omega_{2}+\bar{\omega}_{2}\right)^{2}\left(\omega_{3}+\bar{\omega}_{2}\right)^{2}\left(\omega_{2}+\bar{\omega}_{3}\right)^{2}\left(\bar{\omega}_{3}+\omega_{3}\right)^{2}}, \\
& \eta_{3}=\frac{\alpha \Lambda\left(\omega_{1}-\omega_{2}\right)^{2}}{c_{1}\left(\omega_{1}+\bar{\omega}_{3}\right)^{2}\left(\bar{\omega}_{3}+\omega_{2}\right)^{2}} \text {, } \\
& \rho_{4}=\frac{\alpha^{2} \Gamma\left(\omega_{1}-\omega_{2}\right)^{2}\left(\bar{\omega}_{1}-\bar{\omega}_{3}\right)^{2}}{c_{1}^{2} K_{+} \bar{K}_{+}\left(\omega_{1}+\bar{\omega}_{1}\right)^{2}\left(\omega_{2}+\bar{\omega}_{1}\right)^{2}\left(\omega_{1}+\bar{\omega}_{3}\right)^{2}\left(\bar{\omega}_{2}+\omega_{3}\right)^{2}} \text {, } \\
& \eta_{4}=\frac{\alpha \Lambda\left(\omega_{1}-\omega_{3}\right)^{2}}{c_{1}\left(\omega_{1}+\bar{\omega}_{1}\right)^{2}\left(\bar{\omega}_{1}+\omega_{3}\right)^{2}} \text {, } \\
& \rho_{5}=\frac{\alpha^{2} \Gamma\left(\omega_{1}-\omega_{2}\right)^{2}\left(\bar{\omega}_{2}-\bar{\omega}_{3}\right)^{2}}{c_{1}^{2} K_{+} \bar{K}_{+}\left(\omega_{1}+\bar{\omega}_{2}\right)^{2}\left(\omega_{2}+\bar{\omega}_{2}\right)^{2}\left(\omega_{1}+\bar{\omega}_{3}\right)^{2}\left(\bar{\omega}_{3}+\omega_{2}\right)^{2}}, \\
& \eta_{5}=\frac{\alpha \Lambda\left(\omega_{1}-\omega_{3}\right)^{2}}{c_{1}\left(\omega_{1}+\bar{\omega}_{2}\right)^{2}\left(\bar{\omega}_{2}+\omega_{3}\right)^{2}}, \\
& \rho_{6}=\frac{\alpha^{2} \Gamma\left(\omega_{1}-\omega_{3}\right)^{2}\left(\bar{\omega}_{2}-\bar{\omega}_{1}\right)^{2}}{c_{1}^{2} K_{+} \bar{K}_{+}\left(\omega_{1}+\bar{\omega}_{1}\right)^{2}\left(\omega_{3}+\bar{\omega}_{2}\right)^{2}\left(\omega_{1}+\bar{\omega}_{2}\right)^{2}\left(\omega_{3}+\bar{\omega}_{2}\right)^{2}},
\end{aligned}
$$




$$
\begin{aligned}
& \eta_{6}=\frac{\alpha \Lambda\left(\omega_{3}-\omega_{2}\right)^{2}}{c_{1}\left(\omega_{3}+\bar{\omega}_{1}\right)^{2}\left(\bar{\omega}_{1}+\omega_{2}\right)^{2}}, \\
& \rho_{7}=\frac{\alpha^{2} \Gamma\left(\omega_{1}-\omega_{3}\right)^{2}\left(\bar{\omega}_{2}-\bar{\omega}_{3}\right)^{2}}{c_{1}^{2} K_{+} \bar{K}_{+}\left(\omega_{1}+\bar{\omega}_{2}\right)^{2}\left(\omega_{3}+\bar{\omega}_{2}\right)^{2}\left(\omega_{1}+\bar{\omega}_{3}\right)^{2}\left(\omega_{3}+\bar{\omega}_{3}\right)^{2}} \\
& \eta_{7}=\frac{\alpha \Lambda\left(\omega_{2}-\omega_{3}\right)^{2}}{c_{1}\left(\omega_{2}+\bar{\omega}_{2}\right)^{2}\left(\bar{\omega}_{2}+\omega_{3}\right)^{2}} \\
& \rho_{8}=\frac{\alpha^{2} \Gamma\left(\omega_{2}-\omega_{3}\right)^{2}\left(\bar{\omega}_{1}-\bar{\omega}_{2}\right)^{2}}{c_{1}^{2} K_{+} \bar{K}_{+}\left(\omega_{2}+\bar{\omega}_{1}\right)^{2}\left(\omega_{3}+\bar{\omega}_{1}\right)^{2}\left(\omega_{2}+\bar{\omega}_{2}\right)^{2}\left(\omega_{3}+\bar{\omega}_{2}\right)^{2}} \\
& \eta_{8}=\frac{\alpha \Lambda\left(\omega_{1}-\omega_{3}\right)^{2}}{c_{1}\left(\omega_{1}+\bar{\omega}_{3}\right)^{2}\left(\bar{\omega}_{3}+\omega_{3}\right)^{2}}, \\
& \rho_{9}=\frac{\alpha^{2} \Gamma\left(\omega_{2}-\omega_{3}\right)^{2}\left(\bar{\omega}_{1}-\bar{\omega}_{3}\right)^{2}}{c_{1}^{2} K_{+} \bar{K}_{+}\left(\omega_{2}+\bar{\omega}_{1}\right)^{2}\left(\omega_{3}+\bar{\omega}_{1}\right)^{2}\left(\omega_{2}+\bar{\omega}_{3}\right)^{2}\left(\omega_{3}+\bar{\omega}_{3}\right)^{2}} \\
& \eta_{9}=\frac{\alpha \Lambda\left(\omega_{3}-\omega_{2}\right)^{2}}{c_{1}\left(\omega_{3}+\bar{\omega}_{2}\right)^{2}\left(\bar{\omega}_{3}+\omega_{3}\right)^{2}},
\end{aligned}
$$

where $c_{1}$ and $\alpha$ are real constants.

\section{Competing Interests}

The authors declare that they have no competing interests.

\section{Acknowledgments}

This work has been supported by the Graduate Education Innovation Project of Shanxi Province in China under Grant no. 02100761, by the Special Funds of the National Natural Science Foundation of China under Grant no. 61405137, by the Graduate Education Reform Project in Shanxi Province of China under Grant no. 20132017, by the Department of Science in Shanxi Province of China under Grant no. 2014041035-3, and by the Shanxi Province Science Foundation for Youths under Grant no. 2015021008.

\section{References}

[1] A. Hasegawa and F. Tappert, "Transmission of stationary nonlinear optical pulses in dispersive dielectric fibers. I. Anomalous dispersion," Applied Physics Letters, vol. 23, no. 3, pp. 142-144, 1973.

[2] S. V. Manakov, "On the theory of two-dimensional stationary self-focusing of electromagnetic waves," Soviet Physics-Journal of Experimental and Theoretical Physics, vol. 38, pp. 248-253, 1974.

[3] M. V. Tratnik and J. E. Sipe, "Bound solitary waves in a birefringent optical fiber," Physical Review A, vol. 38, no. 4, pp. 2011-2017, 1988.
[4] C. R. Menyuk, "Pulse propagation in an elliptically birefringent Kerr medium," IEEE Journal of Quantum Electronics, vol. 25, no. 12, pp. 2674-2682, 1989.

[5] V. Achilleos, D. J. Frantzeskakis, P. G. Kevrekidis, and D. E. Pelinovsky, "Matter-wave bright solitons in spin-orbit coupled Bose-Einstein condensates," Physical Review Letters, vol. 110, no. 26, Article ID 264101, 2013.

[6] K. Porsezian and K. Nakkeeran, "Optical solitons in birefringent fibre-Bäcklund transformation approach," Pure and Applied Optics, vol. 6, no. 1, pp. L7-L11, 1997.

[7] A. Hasegawa and Y. Kodama, Solitons in Optical Communication, Oxford University Press, Oxford, UK, 1995.

[8] J. N. Huang, J. Z. Xu, and Y. T. Xiong, Solitons Concepts, Principles and Applications, Higher Education Press, Beijing, China, 2004.

[9] M. J. Ablowitz, Solitons, Nonlinear Evolution Equations and Inverse Scattering, Cambridge University Press, Cambridge, UK, 2004.

[10] M. J. Ablowitz, B. Prinari, and A. D. Trubatch, Discrete and Continuous Nonlinear Schrödinger Systems, Cambridge University Press, Cambridge, Mass, USA, 2003.

[11] A. Ankiewicz, J. M. Soto-Crespo, and N. Akhmediev, "Rogue waves and rational solutions of the Hirota equation," Physical Review E, vol. 81, no. 4, Article ID 046602, 8 pages, 2010.

[12] R. Guo and H.-Q. Hao, "Breathers and multi-soliton solutions for the higher-order generalized nonlinear Schrödinger equation," Communications in Nonlinear Science and Numerical Simulation, vol. 18, no. 9, pp. 2426-2435, 2013.

[13] Y.-J. Feng, Y.-T. Gao, Z.-Y. Sun et al., "Anti-dark solitons for a variable-coefficient higher-order nonlinear Schrödinger equation in an inhomogeneous optical fiber," Physica Scripta, vol. 90, no. 4, Article ID 045201, 2015. 
[14] X. Lü and M. Peng, "Painlevé-integrability and explicit solutions for the general two-coupled nonlinear Schrödinger system in the optical fiber communications," Nonlinear Dynamics, vol. 73, no. 1, pp. 405-410, 2013.

[15] W.-R. Sun, B. Tian, D.-Y. Liu, and X.-Y. Xie, "Nonautonomous matter-wave solitons in a Bose-Einstein condensate with an external potential," Journal of the Physical Society of Japan, vol. 84, no. 7, Article ID 074003, 2015.

[16] L. Wang, Y.-J. Zhu, F.-H. Qi, M. Li, and R. Guo, "Modulational instability, higher-order localized wave structures, and nonlinear wave interactions for a nonautonomous Lenells-Fokas equation in inhomogeneous fibers," Chaos, vol. 25, no. 6, Article ID 063111, 14 pages, 2015.

[17] R. Guo and B. Tian, "Integrability aspects and soliton solutions for an inhomogeneous nonlinear system with symbolic computation," Communications in Nonlinear Science and Numerical Simulation, vol. 17, no. 8, pp. 3189-3203, 2012.

[18] A. H. Bhrawy, M. A. Abdelkawy, and A. Biswas, "Cnoidal and snoidal wave solutions to coupled nonlinear wave equations by the extended Jacobi's elliptic function method," Communications in Nonlinear Science and Numerical Simulation, vol. 18, no. 4, pp. 915-925, 2013.

[19] H.-F. Zhang, H.-Q. Hao, and J.-W. Zhang, "Breathers and soliton solutions for a generalization of the nonlinear Schrödinger equation," Mathematical Problems in Engineering, vol. 2013, Article ID 456864, 5 pages, 2013.

[20] R. Guo and H.-Q. Hao, "Breathers and localized solitons for the Hirota-Maxwell-Bloch system on constant backgrounds in erbium doped fibers," Annals of Physics, vol. 344, pp. 10-16, 2014.

[21] R. Guo, H.-Q. Hao, and L.-L. Zhang, "Dynamic behaviors of the breather solutions for the AB system in fluid mechanics," Nonlinear Dynamics, vol. 74, no. 3, pp. 701-709, 2013.

[22] R. Guo, H.-H. Zhao, and Y. Wang, "A higher-order coupled nonlinear Schrödinger system: solitons, breathers, and rogue wave solutions," Nonlinear Dynamics, vol. 83, no. 4, pp. 24752484, 2016.

[23] J. Li, T. Xu, X.-H. Meng, Y.-X. Zhang, H.-Q. Zhang, and B. Tian, "Lax pair, B acklund transformation and $N$-solitonlike solution for a variable-coefficient Gardner equation from nonlinear lattice, plasma physics and ocean dynamics with symbolic computation," Journal of Mathematical Analysis and Applications, vol. 336, no. 2, pp. 1443-1455, 2007.

[24] S. Rajendran, M. Lakshmanan, and P. Muruganandam, "Matter wave switching in Bose-Einstein condensates via intensity redistribution soliton interactions," Journal of Mathematical Physics, vol. 52, no. 2, Article ID 023515, 16 pages, 2011.

[25] Y.-F. Wang, B. Tian, M. Li, P. Wang, and M. Wang, "Integrability and soliton-like solutions for the coupled higher-order nonlinear Schrödinger equations with variable coefficients in inhomogeneous optical fibers," Communications in Nonlinear Science and Numerical Simulation, vol. 19, no. 6, pp. 1783-1791, 2014.

[26] L. Cveticanin, M. KalamiYazdi, H. Askari, and Z. Saadatnia, "Vibration of a two-mass system with non-integer order nonlinear connection," Mechanics Research Communications, vol. 43, pp. 22-28, 2012.

[27] B. Ge, "Multiple solutions of nonlinear Schrödinger equation with the fractional Laplacian," Nonlinear Analysis: Real World Applications, vol. 30, pp. 236-247, 2016.

[28] J. Xu, Z. Wei, and W. Dong, "Existence of weak solutions for a fractional Schrödinger equation," Communications in Nonlinear
Science and Numerical Simulation, vol. 22, no. 1-3, pp. 1215-1222, 2015.

[29] X.-Y. Xie, B. Tian, W.-R. Sun, and Y. Sun, "Bright solitons for the $(2+1)$-dimensional coupled nonlinear Schrödinger equations in a graded-index waveguide," Communications in Nonlinear Science and Numerical Simulation, vol. 29, no. 1-3, pp. 300-306, 2015.

[30] J. Chai, B. Tian, H.-L. Zhen, and W.-R. Sun, "Lax pair, conservation laws and solitons for a (2+1)-dimensional fourthorder nonlinear Schrödinger equation governing an $\alpha$-helical protein," Annals of Physics, vol. 362, pp. 671-683, 2015.

[31] H.-L. Zhen, B. Tian, Y.-F. Wang, and D.-Y. Liu, "Soliton solutions and chaotic motions of the Zakharov equations for the Langmuir wave in the plasma," Physics of Plasmas, vol. 22, no. 3, Article ID 032307, 2015.

[32] X.-Y. Xie, B. Tian, W.-R. Sun, M. Wang, and Y.-P. Wang, "Solitary wave and multi-front wave collisions for the Bogoyavlenskii-Kadomtsev-Petviashili equation in physics, biology and electrical networks," Modern Physics Letters B, vol. 29, no. 31, Article ID 1550192, 12 pages, 2015.

[33] X.-Y. Gao, "Variety of the cosmic plasmas: general variablecoefficient Korteweg-de Vries-Burgers equation with experimental/observational support," Europhysics Letters, vol. 110, no. 1, Article ID 15002, 2015.

[34] X.-Y. Gao, "Comment on "Solitons, Bäcklund transformation, and Lax pair for the (2+1)-dimensional Boiti-Leon-Pempinelli equation for the water waves" [J. Math. Phys. 51, 093519 (2010)]," Journal of Mathematical Physics, vol. 56, no. 1, Article ID 014101, 2015.

[35] X.-Y. Gao, "Bäcklund transformation and shock-wave-type solutions for a generalized (3+1)-dimensional variablecoefficient B-type Kadomtsev-Petviashvili equation in fluid mechanics," Ocean Engineering, vol. 96, pp. 245-247, 2015.

[36] X.-Y. Gao, "Incompressible-fluid symbolic computation and Bäcklund transformation: $(3+1)$-dimensional variablecoefficient Boiti-Leon-Manna-Pempinelli model," Zeitschrift für Naturforschung A, vol. 70, no. 1, pp. 59-61, 2015.

[37] R. Hirota, The Direct Method in Soliton Theory, Cambridge University Press, Cambridge, UK, 2004.

[38] A.-M. Wazwaz, "The Hirota's direct method for multiple-soliton solutions for three model equations of shallow water waves," Applied Mathematics and Computation, vol. 201, no. 1-2, pp. 489-503, 2008.

[39] Y.-F. Wang, B. Tian, M. Li, P. Wang, and Y. Jiang, "Soliton dynamics of a discrete integrable Ablowitz-Ladik equation for some electrical and optical systems," Applied Mathematics Letters, vol. 35, pp. 46-51, 2014.

[40] X.-d. Yang and H.-y. Ruan, "HBFGen: a maple package to construct the Hirota bilinear form for nonlinear equations," Applied Mathematics and Computation, vol. 219, no. 15, pp. 8018-8025, 2013. 


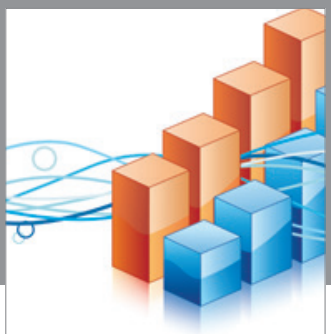

Advances in

Operations Research

vatem alat4

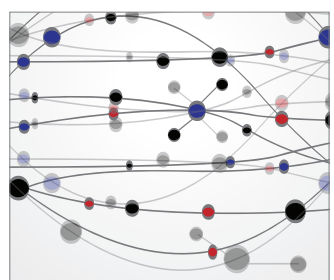

\section{The Scientific} World Journal
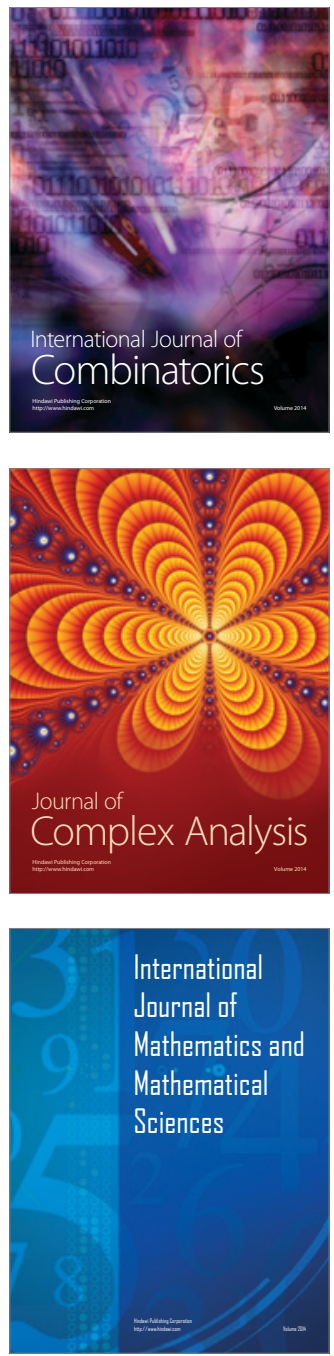
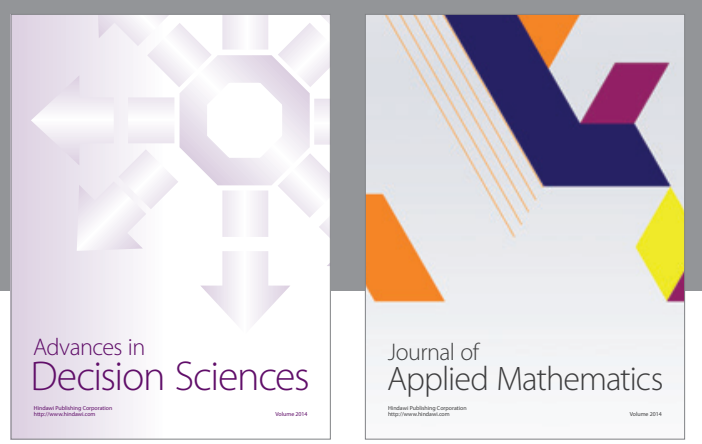

Algebra

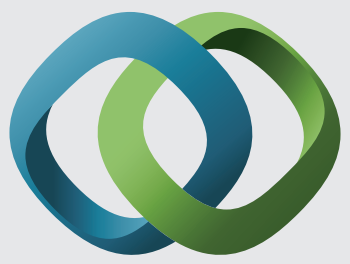

\section{Hindawi}

Submit your manuscripts at

http://www.hindawi.com
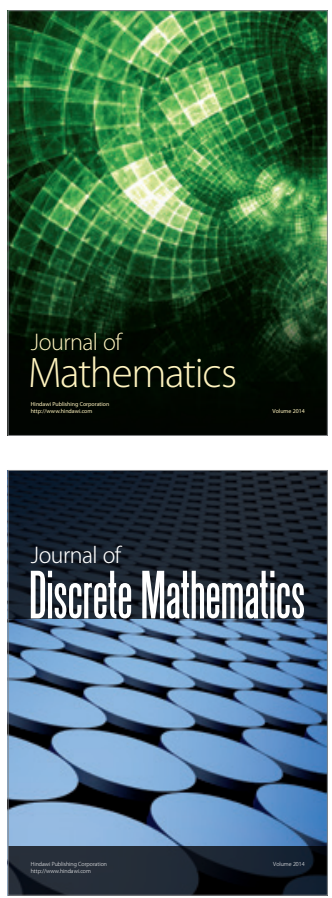

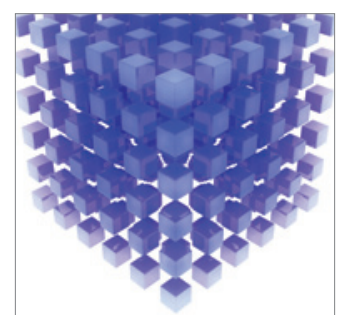

Mathematical Problems in Engineering
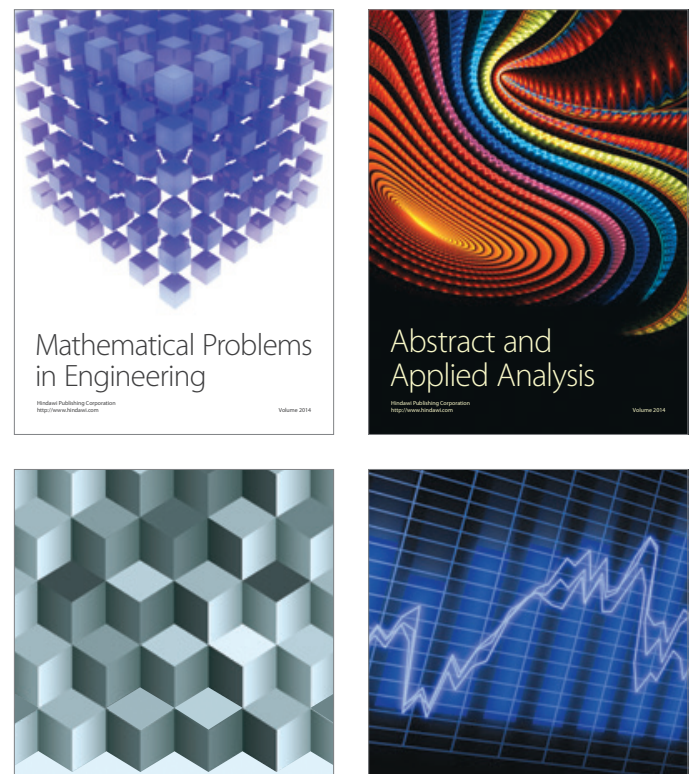

Journal of

Function Spaces

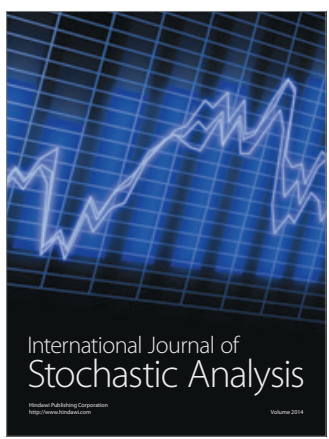

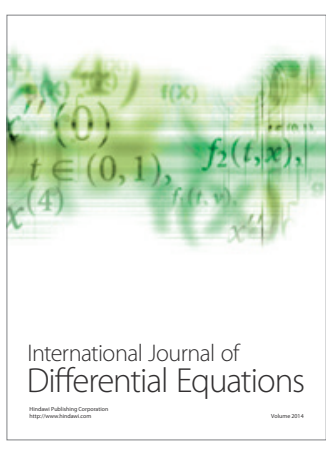
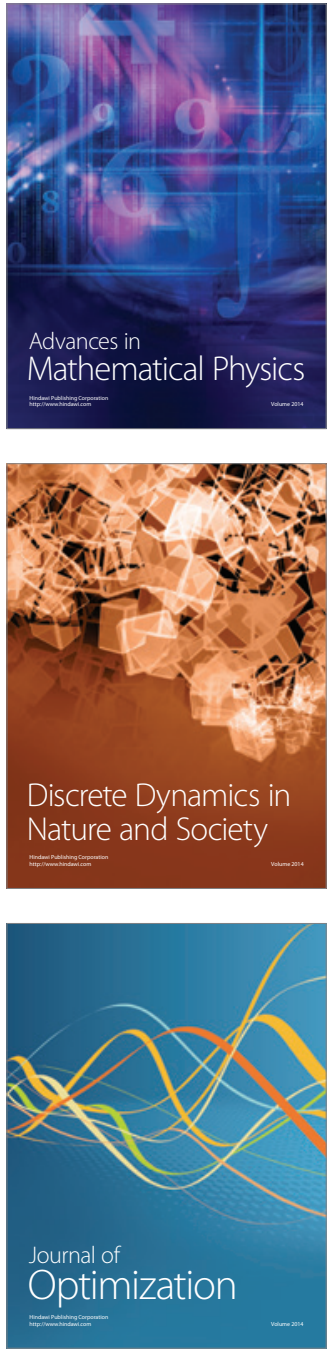